\title{
High-Spectral Resolution Remote Sensing of Soil Organic Carbon Dynamics
}

\author{
Bas van Wesemael ${ }^{1, *(\mathbb{D})}$, Sabine Chabrillat ${ }^{2}$ and Florian Wilken ${ }^{3,4}$ \\ 1 Georges Lemaître Centre for Earth and Climate Research, Earth and Life Institute, \\ Université Catholique de Louvain, 1348 Louvain-la-Neuve, Belgium \\ 2 Remote Sensing and Geoinformatics, Helmholtz Centre Potsdam, GFZ German Research Centre for \\ Geosciences, 14473 Potsdam, Germany; sabine.chabrillat@gfz-potsdam.de \\ 3 Department of Environmental Systems Science, Eidgenössische Technische Hochschule Zürich (ETH), \\ 8092 Zürich, Switzerland; florian.wilken@usys.ethz.ch \\ 4 Institute for Geography, Universität Augsburg, 86159 Augsburg, Germany \\ * Correspondence: bas.vanwesemael@uclouvain.be
}

\section{check for} updates

Citation: van Wesemael, B.; Chabrillat, S.; Wilken, F. HighSpectral Resolution Remote Sensing of Soil Organic Carbon Dynamics.

Remote Sens. 2021, 13, 1293.

https://doi.org/10.3390/rs13071293

Received: 22 January 2021

Accepted: 25 March 2021

Published: 29 March 2021

Publisher's Note: MDPI stays neutral with regard to jurisdictional claims in published maps and institutional affiliations.

Copyright: (c) 2021 by the authors. Licensee MDPI, Basel, Switzerland. This article is an open access article distributed under the terms and conditions of the Creative Commons Attribution (CC BY) license (https:// creativecommons.org/licenses/by/ $4.0 /)$.
Soil organic matter (SOM) is essential for preserving a healthy soil that provides good soil structure and high fertility and water -holding capacity. Furthermore, an SOM increase, and thus its main component, soil organic carbon (SOC), leads to a sequestration of atmospheric $\mathrm{CO}_{2}$ and it is proposed as a nature-based solution to mitigate climate change, which has received great political attention (e.g., Lima Paris Action Agenda: 4\%o initiative; Kyoto protocol: Article 3.4). However, croplands have suffered for centuries from a lack of biomass return to the soil leading to SOC depletion that limits the sequestration potential of soils. To understand SOC dynamics, large spatial heterogeneity needs to be addressed, which typically requires large amounts of soil sample analysis. Visible, near, and shortwave infrared (VNIR-SWIR) soil reflectance spectroscopy is now an accepted alternative to wet chemistry for efficiently processing the large number of samples required to investigate patterns in SOC and monitor its dynamics using proximal and remote sensing. Pilot studies have demonstrated the potential of airborne remote sensing for mapping SOC in the homogenized topsoil of exposed croplands (in particular when they are just seeded) using spectroscopic techniques analogous to the ones used in the laboratory. The development of miniature sensors mounted on unmanned aerial vehicles (UAVs), as well as the high-spectral resolution imaging spectrometers onboard airborne or satellite platforms, have bridged the gap between high-resolution plot and moderate-resolution landscape scale assessments. UAVs are cost-efficient and easy to operate, whereas airborne campaigns are costly to organize and involve heavy infrastructure, although none of them can cover large areas. Satellites such as the Sentinel 2 (S2) multispectral instrument provide a global cover at five-day intervals with lower spectral resolution, whereas recent or upcoming hyperspectral satellites such as PRISMA and EnMAP have higher spectral resolution but limited revisit capabilities. Obviously, the conditions for predicting SOC in croplands from remote sensing platforms are less favorable than for analyzing samples in a laboratory due to the lower spectral resolution, atmospheric disturbance, and heterogeneity of the soil surface (e.g., moisture content, partial crop or residue cover, roughness). However, the potential of mapping SOC patterns from the field to the regional scale and monitoring SOC dynamics over time is unprecedented. This will, without doubt, greatly contribute to the transition to a sustainable and high carbon sequestration agriculture.

The prediction of soil properties, such as SOC, is complex due to the variable spectral response of organic matter, resulting in a lack of clear and narrow spectral features. This Special Issue aims to present the most recent methodological developments and science use cases in improving the quantification of SOC based on VNIR-SWIR spectroscopy data acquired from proximal, unmanned aerial system (UAS), airborne, and satellite highspectral resolution instruments. The papers in this issue demonstrate the potential of 
remote sensing for SOC mapping and monitoring, and address the challenges that need to be resolved before we can establish a (pre-)operational remote sensing SOC mapping and monitoring system. We can subdivide these challenges into the following categories: (i) characterization of soil organic matter composition [1] and related soil properties [2], (ii) soil surface conditions that disturb the spectral signal of the soil [3-6], and (iii) spectral modeling issues from the signal pretreatment [7], from the degradation in spatial and spectral resolution [8], or from using multiple linear regressions including topographic covariates [9] and using soil spectral libraries as calibration data [10].

Soil organic matter is a heterogeneous compound and its characterization by spectral techniques is not always straightforward. One of the challenges is the partial overlap of organic spectral features with hematite iron oxide features in the VNIR spectral range (400-1000 nm). Heller Pearlshtien and Ben Dor [1] investigate the effect of different SOM species in Mediterranean soils rich in hematite iron oxides. They found out that synthetic mixture could not imitate the authentic soil reflectance status, especially across the overlapping spectral position of the iron oxides and $\mathrm{OM}$, and hence may hinder real conditions. They emphasize that saturation effects of SOM on the iron oxide VNIR spectral signal should be taken into account when iron oxide and SOM contents are intended to be individually estimated. For the next study on aggregate stability, it is well-known that SOM plays a dominant role in aggregate stability and even more for cropland soils that are generally poor in organic matter. The quantification of aggregate stability involves laborious techniques such as wet sieving and particle size analysis to derive parameters such as the mean weight diameter (MWD) of water stable aggregates. It is therefore not surprising that mapping and monitoring of aggregate stability is hardly feasible in practice. Shi et al. [2] propose to build on the correlation of MWD and SOC, allowing them to map aggregate stability for croplands in the flight strip of an airborne hyperspectral sensor.

The spectra of cropland soils in seedbed condition reflect in ideal conditions the spectra of an air-dried and sieved sample, as one can find in a soil spectral library. However, in less ideal conditions, moisture, partial crop, and residue cover, as well as shading by soil clods, can disturb the signal from the soil surface, because of which spectra from remote sensors cannot be compared to the ones of the soil spectral library anymore. The following studies deal with these limitations. Nawar et al. [3] demonstrate how spectra acquired from an online prediction system can be efficiently corrected for soil moisture effects using the external parameter orthogonalization (EPO) approach. Such systems are used in precision agriculture, and the spectra are acquired from a tine pulled through the topsoil by a tractor. The EPO approach requires a set of corresponding moist and air-dried samples that are scanned simultaneously with the same spectrometer, conditions which can mostly be met for proximal sensors mounted onsite. Vaudour et al. [4] investigate the performance of SOC prediction models for S2 images acquired at different dates. They acknowledge that the optimal conditions for SOC prediction are rare on a single S2 image and that the SOC content can only be predicted in a fraction of the cropland area. The main factors influencing the performance of the SOC prediction models were soil roughness in conjunction with soil moisture and the cloud and shadow cover over the entire tile. They show that the best performing dates for S2 SOC mapping were spring dates and that NDVI values below 0.35 did not influence prediction performance, opening perspectives for further use of Sentinel-2 into multidate mosaics. Castaldi et al. [5] used the European-wide LUCAS spectral library for the calibration of an SOC prediction model for an entire S2 tile acquired in late summer in Northern Germany. They advocate that the Normalized Burn Ratio 2 (NBR2), based on the two SWIR bands of S2 (B11 and B12), can be used to mask pixels influenced by moisture content and/or crop residues, and investigate different NBR2 thresholds. Dvorakova et al. [6] further investigate the potential of the NBR2 index. The authors compare the NBR2 index based on a hyperspectral airborne campaign and S2 images under dry conditions in late summer, and additionally for an S2 scene in wet condition in autumn. The higher spectral resolution of the airborne sensor allowed the cellulose absorption index (CAI) to be calculated in the SWIR. The CAI proved 
to be efficient in masking pixels with a partial crop residue cover and correlated well with the NBR2 index in dry conditions, but unfortunately not for wet conditions. The new generation of hyperspectral satellites (e.g., PRISMA, EnMAP, later CHIME, SBG, etc.) will certainly improve the possibilities for correction for residues and soil moisture as the spectral resolution allows for an improved determination of these parameters based on spectral indices or other approaches.

Shen et al. [7] tested 54 different spectral pretreatments to preprocess soil spectral data acquired in the laboratory for an improved SOM prediction using partial least squares regression (PLSR) techniques. These spectral pretreatments consisted of three denoising methods, six data transformations, and three dimensionality reduction methods. Overall, the denoising caused a strong reduction of overfitting and increased accuracy. The pretreatment will become ever more important as remote sensing platforms evolve from multispectral to hyperspectral instruments. On the one hand, multispectral satellite data are now readily available and their frequency of overflight allows the establishment of time series. On the other hand, multispectral and hyperspectral UAV systems are affordable options for acquiring images at the field scale under ideal conditions, as the operators can quickly launch a campaign at low costs. Žížala et al. [8] compared the performance of SOC prediction models in a large cropland field for multispectral satellite and UAV sensors with different spatial and spectral resolution against a baseline using an airborne hyperspectral sensor. A moderately strong spatial correlation was obtained between the baseline SOC maps and the maps produced by all other sensors. Wehrhahn and Sommer [9] provide an example of high-resolution UAV-based SOC mapping in a number of cropland fields in hummocky terrain where tillage erosion creates high spatially-distributed SOC dynamics. Multiple linear regression models including both spectral and topographic variables already produce robust SOC prediction models. For mapping of large areas using satellite imagery, the consistent analysis of calibration samples becomes a bottleneck. Ward et al. [10] used a two-step approach to predict the SOC contents for airborne HySpex and simulated EnMAP imagery acquired in northeastern Germany, based on the local PLSR method for model building. First, the local PLSR uses the European LUCAS soil spectral database to quantify the SOC content for soil samples from the study site, and second, a remote sensing model is calibrated based on the local PLSR SOC results and the corresponding image spectra.

This special issue demonstrates that the field of SOC prediction from remote sensing is evolving rapidly thanks to the developments in soil databases, sensor technology (UAV, airborne, spaceborne), modeling techniques, and to the advancing recognition of the urge for global monitoring of soils. High-spatial resolution satellite data such as Sentinel 2 are now readily available at five-day intervals and the miniature spectrometers suitable for UAV platforms have become an affordable technology. Similarly, airborne platforms have become more and more accessible, and upcoming spaceborne platforms are planned and more will be available in the near future. Technical and methodological developments will continue and upcoming high-quality spectroscopy data and tools for soil analyses based on spectral databases will become more and more available, and applicable for all types of platforms such as in the laboratory, onsite mounted on tractors, from UAV, and air- and spaceborne platforms. This will open new perspectives for improved and more regular SOC mapping and for supporting the monitoring of SOC stocks for which an urgent demand exists in the framework of climate change mitigation.

Funding: This research received no external funding.

Conflicts of Interest: The authors declare no conflict of interest.

\section{References}

1. Heller Pearlshtien, D.; Ben-Dor, E. Effect of Organic Matter Content on the Spectral Signature of Iron Oxides across the VIS-NIR Spectral Region in Artificial Mixtures: An Example from a Red Soil from Israel. Remote Sens. 2020, 12, 1960. [CrossRef] 
2. Shi, P.; Castaldi, F.; van Wesemael, B.; Van Oost, K. Large-Scale, High-Resolution Mapping of Soil Aggregate Stability in Croplands Using APEX Hyperspectral Imagery. Remote Sens. 2020, 12, 666. [CrossRef]

3. Nawar, S.; Abdul Munnaf, M.; Mouazen, A.M. Machine Learning Based On-Line Prediction of Soil Organic Carbon after Removal of Soil Moisture Effect. Remote Sens. 2020, 12, 1308. [CrossRef]

4. Vaudour, E.; Gomez, C.; Loiseau, T.; Baghdadi, N.; Loubet, B.; Arrouays, D.; Ali, L.; Lagacherie, P. The Impact of Acquisition Date on the Prediction Performance of Topsoil Organic Carbon from Sentinel-2 for Croplands. Remote Sens. 2019, 11, 2143. [CrossRef]

5. Castaldi, F.; Chabrillat, S.; Don, A.; van Wesemael, B. Soil Organic Carbon Mapping Using LUCAS Topsoil Database and Sentinel-2 Data: An Approach to Reduce Soil Moisture and Crop Residue Effects. Remote Sens. 2019, 11, 2121. [CrossRef]

6. Dvorakova, K.; Shi, P.; Limbourg, Q.; van Wesemael, B. Soil Organic Carbon Mapping from Remote Sensing: The Effect of Crop Residues. Remote Sens. 2020, 12, 1913. [CrossRef]

7. Shen, L.; Gao, M.; Yan, J.; Li, Z.-L.; Leng, P.; Yang, Q.; Duan, S.-B. Hyperspectral Estimation of Soil Organic Matter Content using Different Spectral Preprocessing Techniques and PLSR Method. Remote Sens. 2020, 12, 1206. [CrossRef]

8. Žižăala, D.; Minařík, R.; Zádorová, T. Soil Organic Carbon Mapping Using Multispectral Remote Sensing Data: Prediction Ability of Data with Different Spatial and Spectral Resolutions. Remote Sens. 2019, 11, 2947. [CrossRef]

9. Wehrhan, M.; Sommer, M. An efficient approach to estimate soil organic carbon applying Unmanned Aerial System (UAS) multispectral imagery and the Topographic Position Index in a heterogeneous soil landscape. Remote Sens. 2021, in press.

10. Ward, K.J.; Chabrillat, S.; Brell, M.; Castaldi, F.; Spengler, D.; Foerster, S. Mapping Soil Organic Carbon for Airborne and Simulated EnMAP Imagery Using the LUCAS Soil Database and a Local PLSR. Remote Sens. 2020, 12, 3451. [CrossRef] 